\title{
Optimal Plane Changes Using Third-Body Forces
}

\author{
B.F. VILLAC AND D.J. SCHEERES \\ Department of Aerospace Engineering, The University of Michigan, \\ Ann Arbor, Michigan, USA
}

\begin{abstract}
The fuel optimality of third-body driven plane changes (i.e., plane changes performed by using third-body forces) over one-impulse transfers is investigated numerically and analytically. In particular, the range of thirdbody driven plane changes that are realizable is shown to be restricted and one impulse must be used in the uncovered regions. However, when third-body driven plane changes are realizable, it is shown that they are always optimal above a certain critical value (about $40^{\circ}$ ) that depends on the initial condition. Contour plots of optimal $\Delta V$ values to perform a desired plane changes are given.
\end{abstract}

KEYwORDS: plane change maneuvers; third-body dynamics; Hill's problem

\section{INTRODUCTION}

For low Earth orbiters, small plane change maneuvers are based on a classical approach using the two-body problem (2BP) as the underlying model. During one orbit, third-body perturbations result in little effect and 2BP model analysis yields estimates of the cost of such a maneuver that are sufficiently good for the preliminary design phase. ${ }^{1}$ However, for large plane changes (larger than $60^{\circ}$ ), the classical optimal solution is to place the spacecraft on a parabolic transfer trajectory and perform the plane change "at infinity". In addition to the infeasibility of such an approach (which requires an infinite transfer time), approximate implementation of the strategy by raising the apoapsis radius of the transfer ellipse can no longer be well described by the 2BP, because of the larger Luni-Solar perturbations that result from the increase of the apoapsis radius. ${ }^{2}$ The $2 \mathrm{BP}$ analysis no longer yields estimates of the cost associated with a plane change maneuver that are accurate enough to be useful.

This situation is especially prevalent in environments strongly perturbed by a third body; that is, when the gravitational perturbation of a third body is the dominant perturbation (as, for example, in the environment of a moon of a giant planet), where the only classical approach possible for performing plane changes is the one-impulse plane change maneuver. This method is, however, very expensive and a new class of plane change maneuvers that may be more optimal (depending on the initial conditions) has been devised. ${ }^{3}$ These transfers, third-body driven plane changes, are

Address for correspondence: D.J. Scheeres, Department of Aerospace Engineering, The University of Michigan, Ann Arbor, MI 48109-2140, USA.

scheeres@umich.edu

Ann. N.Y. Acad. Sci. 1017: 255-266 (2004). (C2004 New York Academy of Sciences. doi: 10.1196/annals.1311.015 
based on classical bielliptic plane change maneuvers, where the apoapsis maneuver is suppressed by the use of cost-free third-body forces. The aim of this paper is to investigate the optimality of such transfers in some detail, by answering the question: given a spacecraft on a circular orbit (with given radius $r$ and inclination $i$ ), what is the most cost effective approach to perform a desired plane change maneuver $\Delta i$ ?

Although the previous analysis of third-body driven plane changes searches for the global extremum of plane change that are realizable for given initial conditions, ${ }^{3}$ the answer to the above question requires a closer look at the range of the possible plane changes that are realizable using third-body forces. This range does not cover the set of all allowable plane changes, and one-impulse maneuvers must still sometimes be used. In the case of third-body driven plane changes, analytic estimates and a numerical algorithm are proposed to compare third-body transfers and direct oneimpulse maneuvers. It is shown, in particular, that third-body driven plane changes are always optimal above a critical value (about $40^{\circ}$ ) that depends on the initial conditions. The resulting criterion for optimality allows us to directly choose the optimal approach for a plane change maneuver for given initial conditions.

\section{THIRD-BODY DRIVEN PLANE CHANGES}

The plane change problem can be formulated as that of finding a trajectory that transfers a spacecraft on an initial circular orbit of given radius $r_{0}$ and inclination $i$ to a final circular orbit with same radius $r_{0}$ but with a different inclination, $i+\Delta i$. Although perfectly circular orbit do not exit in environments perturbed by a third body, quasicircular trajectories exist and can be approximated by circular orbits for low altitude and relatively short time span (several revolutions), modulo a precession of the longitude of the ascending node. ${ }^{4}$ In this framework, the plane change problem continues to make sense.

After briefly presenting the underlying model for the dynamics we summarize the results obtained in Villac and Scheeres. ${ }^{3}$

\section{Hill's Problem}

In a restricted two-body problem the center of mass of the attracting body can be taken as the coordinate of an inertial reference frame, however, third-body forces result in setting the attracting masses in motion. The resulting equations of motion are therefore time dependent in inertial space, but can be set to an autonomous form in a rotating frame (assuming circular motion of the primaries around each other). The restricted three-body problem (R3BP) is an example of such a formulation and is widely used in many problems. Additional simplifications of the equations of motion can be performed when one is interested only in the dynamics close to one body. This simplification leads to the Hill equations. They are formulated in a rotating frame centered at the center of mass of the attracting body of interest (see FIGURE 1).

As well as rotation of the frame, the third-body forces result in linear gravitation perturbations that depend on the position of the small body (spacecraft). These equations can be expressed as follows: 


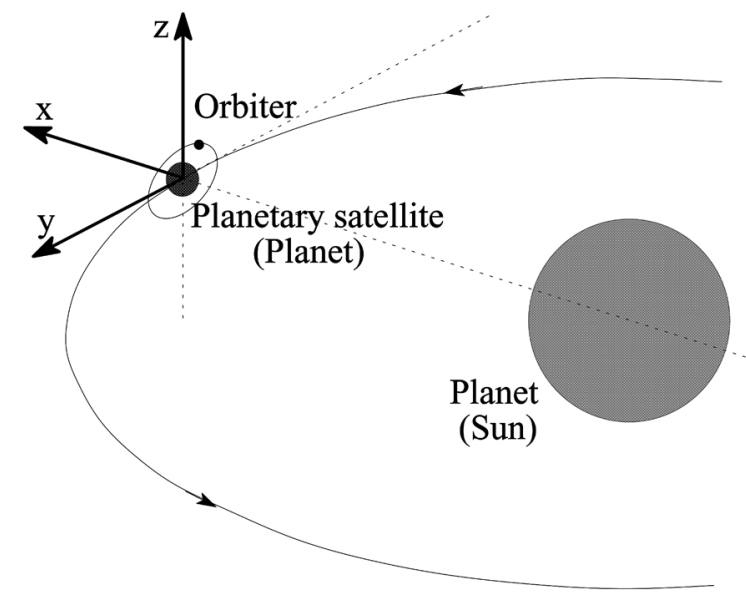

FIGURE 1. Geometry of the Hill problem in the case of an orbiter.

$$
\begin{gathered}
\ddot{x}-2 \dot{y}=-\frac{x}{r^{3}}+3 x \\
\ddot{y}+2 \dot{x}=-\frac{y}{r^{3}} \\
\ddot{z}=-\frac{z}{r^{3}}-z,
\end{gathered}
$$

where the unit length and time scale have been set to $l=\left(\mu / N^{2}\right)^{1 / 3}$ and $\tau=1 / N$, respectively, with $N$ denoting the angular velocity of the frame and $\mu$ denoting the gravitational parameter of the attracting body.

These equations are non-integrable but admit a first integral of motion, known as the Jacobi constant,

$$
J=\frac{1}{2} v^{2}-\frac{1}{r}-\frac{1}{2}\left(3 x^{2}-z^{2}\right)
$$

where $r$ and $v$ denote, respectively, the magnitudes of the radius and velocity vectors of the spacecraft. In addition to the restrictions it imposes on the motion of the spacecraft, this constant is used to analyze the limit of optimality between third-body driven and one-impulse plane changes.

Another feature of the Hill equations is the presence of symmetries. In particular, the following symmetries

$$
\begin{array}{ll}
(x, y, z, \dot{x}, \dot{y}, \dot{z}, t) & \stackrel{S_{1}}{\rightarrow}(-x,-y, z,-\dot{x},-\dot{y}, \dot{z}, t) \\
(x, y, z, \dot{x}, \dot{y}, \dot{z}, t) & \stackrel{S_{2}}{\rightarrow}(x, y,-z, \dot{x}, \dot{y},-\dot{z}, t)
\end{array}
$$

correspond to the invariance of the dynamics by shifts of $m \pi(m=0, \pm 1, \ldots)$ in argument of periapsis $(\omega)$ and longitude of the ascending node as measured from the positive $x$-axis $(\Omega)$, respectively. Therefore, analysis depending on $\omega$ and $\Omega$ can be restricted to the space $\left[0,180^{\circ}\right] \times\left[0,180^{\circ}\right]$ for these variables. 


\section{Classic versus Third-Body Plane Changes}

One-impulse plane changes consist of rotating the velocity vector by $\Delta i$ at one of the nodes of the initial circular orbit. The change in velocity needed to perform such a transfer is given by

$$
\Delta V=2 V_{l c} \sin \left(\frac{|\Delta i|}{2}\right)
$$

where $V_{l c}=1 / r$ represents the local circular speed in inertial space. This transfer is optimal in a two-body framework for plane changes smaller than $48^{\circ} .^{1}$ For larger plane changes, the classical approach would be to first raise the apoapsis radius of the transfer ellipse and then perform the plane change maneuver at apoapsis before recircularizing the trajectory at the next periapsis. This last approach is no longer valid in a three-body framework since changes in orbital elements occur between two successive periapsis passages. However, the forces causing these changes can be controlled by choosing $\omega$ and $\Omega$ of the transfer trajectory to obtain "cost free" plane changes over a single revolution.

More precisely, third-body driven plane change consists of applying a tangential impulsive maneuver at a precise instant so that the desired plane change is performed by the third-body forces along the transfer trajectory. The choice of the time of the maneuver also insures a zero change in periapsis radius over the transfer trajectory so that a second maneuver at the next periapsis can insert the spacecraft in the final circular orbit (see FIGURE 2).

The choice of the time of the maneuver is determined by the choice of $\omega$ and $\Omega$ of the transfer trajectory. Indeed, the time of the maneuver determines the elements of the transfer trajectory at periapsis. In particular, the mean anomaly of the spacecraft on the initial circular orbit sets the argument of periapsis of the transfer trajectory. The choice of $\omega$ and $\Omega$ is determined by solving an optimization problem corresponding to the plane change problem,

$$
\max _{\omega, \Omega} \Delta i_{(\omega, \Omega)} \text { subject to } \Delta r_{p(\omega, \Omega)}=0,
$$

where the $\Delta$ represents a change between successive periapsis passages.

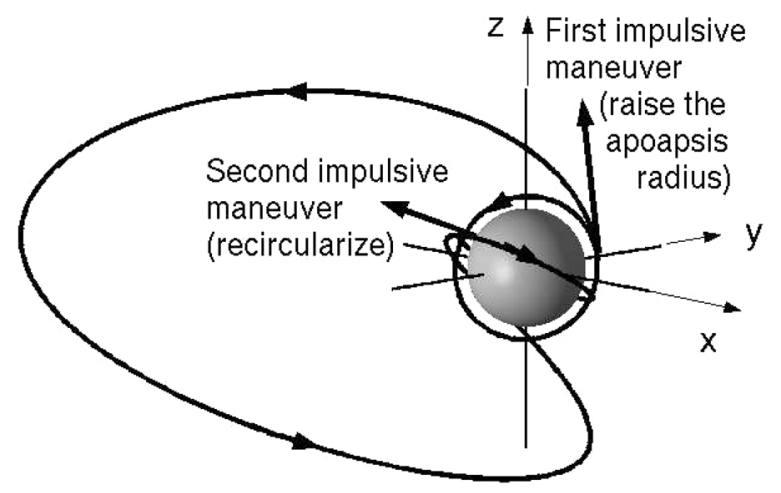

FIGURE 2. Third-body driven plane change. 
Note that, in this optimization problem, $\Delta i$ and $\Delta r_{p}$ are also functions of $r_{p}, r_{a}$, and $i$, but these values are determined by the elements of the initial circular orbit, and $r_{a}$ is set so that the maximum plane change obtained corresponds to the desired value of plane change. It has been shown $^{3}$ that the magnitude of the extremum of $\Delta i$ increases with $r_{a}$.

\section{COMPARISON BETWEEN ONE-IMPULSE AND THIRD-BODY DRIVEN PLANE CHANGES}

It has been shown ${ }^{3}$ that third-body driven plane changes can be less (fuel) costly than one-impulse maneuvers for large plane changes. However, the above formulation does not solve the problem of determining what the optimal approach is for performing a given plane change starting with given initial conditions. Moreover, the precise value of $r_{a}$ that needs to be chosen is not specified in the solution of the above problem, it must be given a priori. In practice, one wants to specify the initial conditions available (radius of the circular orbit, initial and final inclinations) and determine the minimum $\Delta V$ needed to perform such a maneuver. These questions are answered in this section, using both analytic and numerical methods.

\section{Approaches to the Solution}

A natural approach to solve the optimality question is to compare the $\Delta \mathrm{V}$ given by the various methods to perform a fixed, given plane change, $\Delta i_{0}$. For one-impulse maneuvers, this value is determined by Equation (5) and for third-body driven plane changes, one needs to solve the following optimization problem:

$$
\min _{\omega, \Omega} \Delta V_{\left(\omega, \Omega, r_{a}\right)} \text { subject to } \Delta i_{\left(\omega, \Omega, r_{a}\right)}=\Delta i_{0} \text { and } \Delta r_{p\left(\omega, \Omega, r_{a}\right)}=0 \text {. }
$$

Although this approach gives a complete solution to the problem, it is not computationally effective, and problem (7) does not necessarily have a solution for all initial conditions.

Therefore, a better approach consists of first investigating the question of the optimal method to be used without worrying about the precise numerical value of $\Delta V$ required. In particular, a first computation must answer the question about the range of third-body driven plane changes possible. This is done in the next subsection by finding the "local-global" extrema of $\Delta i$.

To solve for the question of the optimal method, the Jacobi constant can be used to obtain rather sharp estimates for the third-body driven $\Delta V$ value needed to perform a given plane change with given initial conditions $r_{p}, r_{a}$, and $i$. These estimates result in an upper bound (a function of $r_{p}, r_{a}, i$, and $\Delta i$ ) above which third-body driven plane changes are shown to be optimal.

These constraints do not, however, solve the problem completely and a numerical algorithm must be used to complete the answer. The idea of the algorithm proposed is based on comparing the plane changes obtained via both methods for a given $\Delta V$ value. This results in reducing the question of the optimal method to that of solving problem (6) instead of (7). These results can then be used to restrict the domain on which one need solve (7) in order to obtain the optimal third-body driven $\Delta V$. 


\section{Realizable Range of Third-Body Driven Plane Changes}

In Reference 3, the solution of (6) was obtained by finding the global extrema of $\Delta i$ on the manifolds $\mathbb{R}=\left\{(\omega, \Omega) \mid \Delta r_{p(\omega, \Omega)}=0\right\}$. Although there is a unique global maximum and minimum for any given values of $r_{p}, r_{a}$, and $i$, their positions in the $(\omega, \Omega)$-space may not be on the same connected component of $\mathcal{Z}$. In such cases, it may happen that the range of third-body driven plane changes between the computed extrema is not connected. This happens generally for near equatorial orbits $\left(i\right.$ near $0^{\circ}$ or $180^{\circ}$ ).

To determine the range of possible third-body driven plane changes, the extrema of $\Delta i$ are computed by pairs on each connected component of $\mathfrak{R}$. Any plane change can then be realized between the two extrema of a given pair.

The connected components of $\mathfrak{R}$ are computed, as described elsewhere, ${ }^{3}$ by first searching along the axis $\omega=0$ and $\Omega=0$, and then by tracking the $\Delta r_{p}=0$ lines step by step along their tangents. FIGURE 3 represents a connected component of $\Delta r_{p}=0$ with the location of the pair of global extrema on this component. All the plane change values between $-60^{\circ}$ and $+39^{\circ}$ are possible in this case.

FIGURE 4 shows the results of the computation of the pairs of $\Delta i$ extrema for $r_{p}=0.003$, at $r_{a}=0.4$ and 0.5 , as a function of inclination. It can be seen that, near the equatorial plane, a large range of values of plane changes are not realizable using third-body driven plane changes and these initial conditions. This is also true for plane change values that correspond to transferring the spacecraft to the equatorial plane. These restrictions are the result of using only the natural dynamics to operate the plane change during the transfer. Indeed, it can be easily checked that the equatorial plane is invariant under the Hill equations.

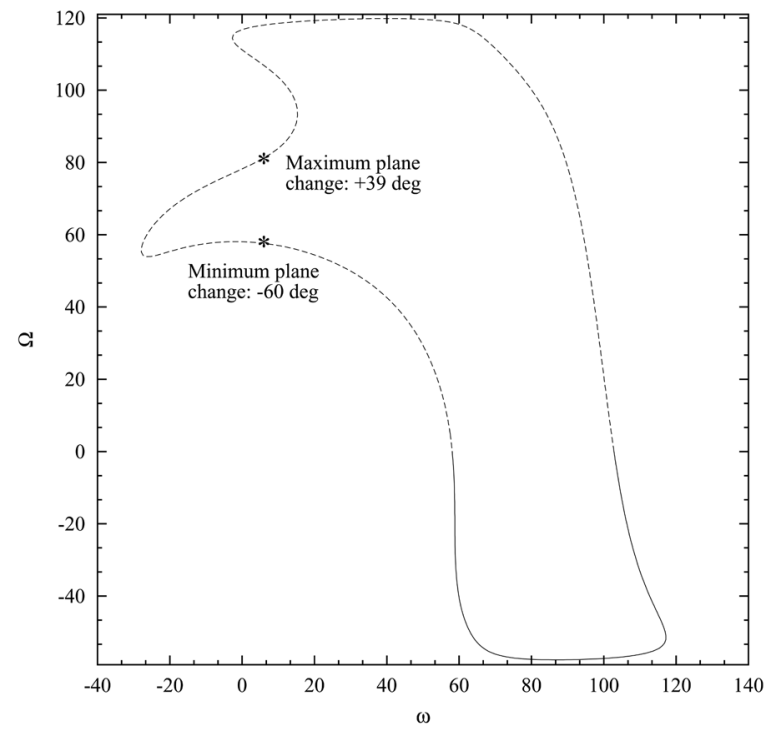

FIGURE 3. $\Delta r_{p}=0$ line for $r_{p}=0.08, r_{a}=0.6$, and $i=90^{\circ}$. 
From a practical point of view, one needs to impose a limit on the maximal apoapsis radius that can be reached, and the range of third-body driven plane changes realizable corresponds to the range obtained with this maximal apoapsis radius. Such a limit may be imposed either by the limitations of the model (the Hill's model is not representative of the dynamics of a spacecraft far from the primary) or by constraints imposed on the maximal time of flight allowed (the larger the apoapsis radius, the longer the time of flight). In this paper we impose $r_{a_{\max }}<0.7$, which corresponds approximately to the distance of the libration points $L_{1,2}$ from the origin. For $r_{p}=0.003$, the realizable range of third-body driven plane changes is shown in FIGURE 5 for $r_{a_{\max }}=0.4$ and 0.5 . These graphs corresponds to the envelopes of the results shown in FIGURE 4.

In terms of comparison with one-impulse plane changes, the above results show that outside the realizable range computed, one-impulse plane changes must be used and are, per force, optimal.
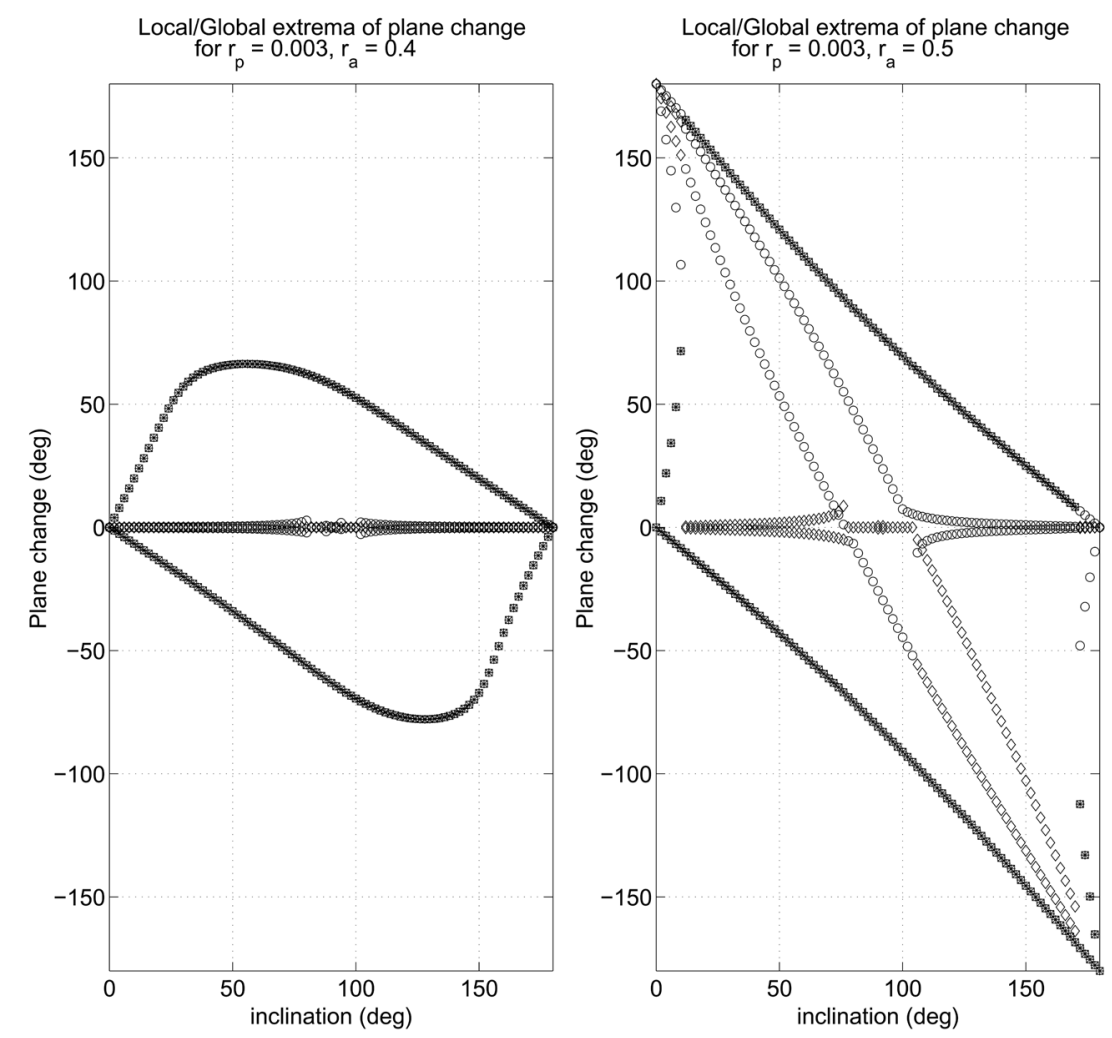

FIGURE 4. Local-global extrema on individual zero lines. 


\section{Optimal Method for Performing a Desired Plane Change}

In the range of possible third-body driven plane changes, the question of the comparison with one-impulse maneuvers must still be answered. To do so, an estimate of $\Delta V_{\text {third-body }}\left(r_{p}, r_{a}, i, \Delta i\right)$ is obtained first, via the Jacobi constant expressed in inertial coordinates

$$
J=\frac{1}{2} v^{2}-\frac{1}{r}-h \cos i+\frac{1}{2} r^{2}-\frac{3}{2} x^{2},
$$

where $r, v$, and $h$ denote, respectively, the magnitude of the position, velocity, and angular momentum vector in inertial space (using the same origin as in the rotating frame).

Along a transfer trajectory, $\omega$ and $\Omega$ were chosen so that $\Delta r_{p}=0$. It follows that the terms depending on $r$ only cancel out in the difference of the Jacobi constant taken between the final and initial periapsis passages of the transfer trajectory. Moreover, at periapsis, the angular momentum magnitude is expressed as $h=r_{p} v$, so that we obtain
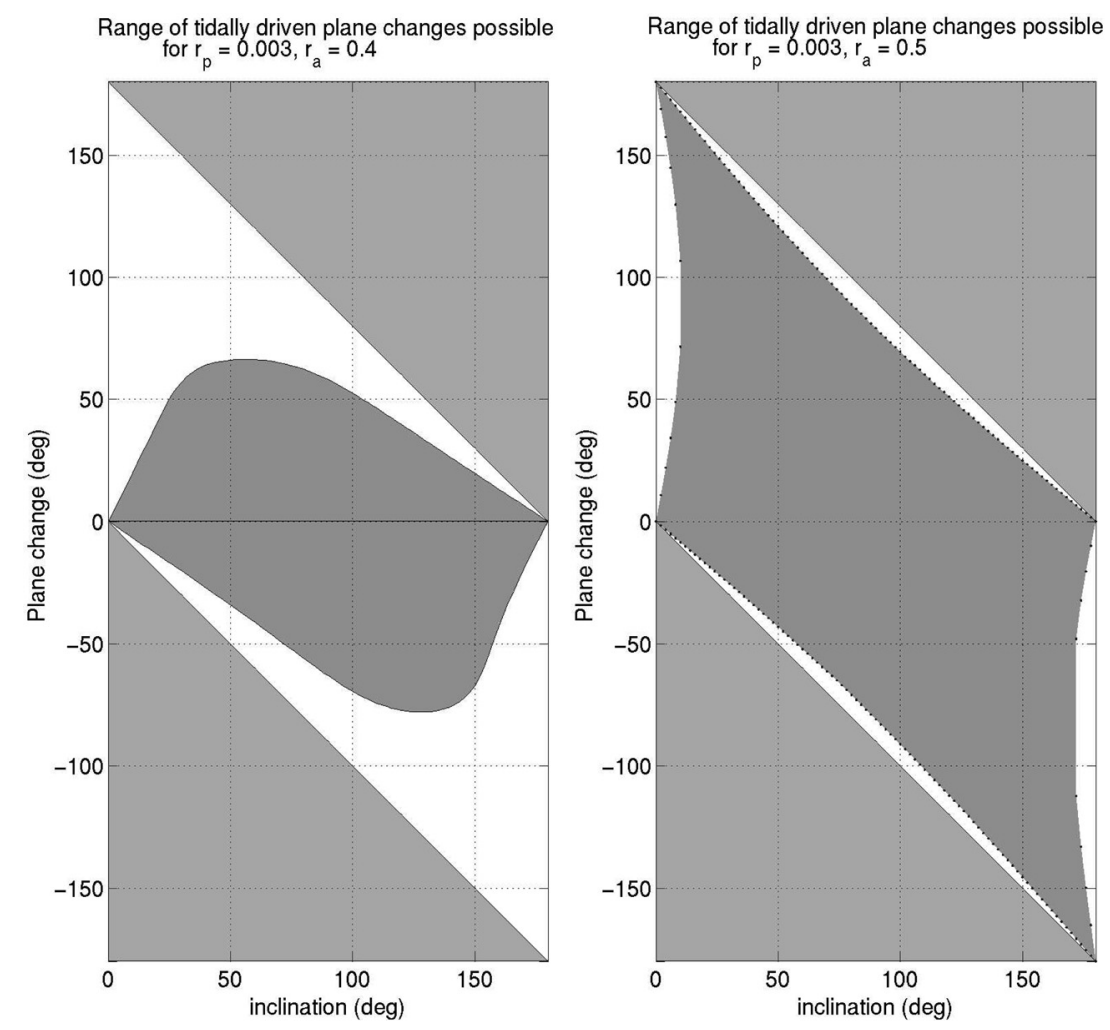

FIGURE 5. Range of possible third-body driven plane changes. 


$$
\begin{aligned}
D J & =J_{2}-J_{1}=0 \\
& =\frac{1}{2}\left(v_{2}^{2}-v_{1}^{2}\right)-r_{p} v_{2} \cos (i+\Delta i)+r_{p} v_{1} \cos (i)-\frac{3}{2}\left(x_{2}^{2}-x_{1}^{2}\right),
\end{aligned}
$$

where the subscripts 1 and 2 refer to the initial and final periapsis, respectively.

Denoting $c=\frac{3}{2}\left(x_{2}^{2}-x_{1}^{2}\right)$, we can solve for $v_{2}$ (quadratic form in $v_{2}$ )

$$
v_{2}=r_{p} \cos (i+\Delta i)+\sqrt{r_{p}^{2} \cos ^{2}(i+\Delta i)+v_{1}^{2}-2 r_{p} v_{1} \cos (i)+2 c} \text {. }
$$

Note here, that $v_{1}$ is expressed in terms of $r_{p}$ and $r_{a}$ of the transfer trajectory at the initial periapsis as $v_{1}=\alpha V_{l c}$ where $\alpha=\sqrt{2 r_{a} /\left(r_{p}+r_{a}\right)}$ depends only on the ratio $r_{a} / r_{p}$. Here $V_{l c}=1 / r_{p}$ is the local circular velocity in inertial space.

The quantity $c$ in (10) depends on $\omega$ and $\Omega$ and can be bounded as

$$
-\frac{3}{2} r_{p}^{2} \cos ^{2}(i) \leq c \leq \frac{3}{2} r_{p}^{2} \cos ^{2}(i+\Delta i),
$$

so that we obtain a bound on $v_{2}$ as follows:

$$
v_{2}^{-} \leq v_{2} \leq v_{2}^{+},
$$

where

$$
\begin{aligned}
& v_{2}^{-}=r_{p} \cos (i+\Delta i)+\sqrt{r_{p}^{2} \cos ^{2}(i+\Delta i)-3 r_{p}^{2} \cos ^{2}(i)+v_{1}^{2}-2 r_{p} v_{1} \cos (i)} \\
& v_{2}^{+}=r_{p} \cos (i+\Delta i)+\sqrt{4 r_{p}^{2} \cos ^{2}(i+\Delta i)+v_{1}^{2}-2 r_{p} v_{1} \cos (i)}
\end{aligned}
$$

It follows that $\Delta V_{\text {third-body }}\left(r_{p}, r_{a}, i, \Delta i\right)=v_{1}+v_{2}-2 V_{l c}$ is also bounded,

$$
\Delta V^{-} \leq \Delta V_{\text {third-body }} \leq \Delta V^{+},
$$

yielding a bound on the limit of optimality for one-impulse plane change maneuvers. Indeed, for any $\Delta i$ in the realizable range, the minimum $\Delta V_{\text {third-body }}$ to perform such plane changes satisfies

$$
\Delta V_{\text {third-body }}(\Delta i) \leq \Delta V^{+}\left(\Delta i, r_{a_{\max }}\right) .
$$

Therefore, it is clear that third-body driven plane changes will be optimal, at least when

$$
\Delta V^{+}\left(\Delta i, r_{a_{\max }}\right) \leq \Delta V_{1-\text { impulse }}(\Delta i) .
$$

FIGURE 6 shows the resulting regions for $r_{p}=0.003$ and $r_{a}=0.5$. For plane changes outside the shaded regions, third-body driven plane changes are optimal when possible (i.e., in the realizable domain).

We also note that the dependence on $i$ and $\Delta i$ in $\Delta V^{+}$is small and the removal of this dependence in (13) leads to an explicit upper limit for optimality of third-body driven plane changes,

$$
|\Delta i| \geq 2 \operatorname{asin}\left\{\frac{\alpha}{2}-1+\frac{r_{p}^{3 / 2}}{2}+\frac{1}{2} \sqrt{\left(\alpha+r_{p}^{3 / 2}\right)^{2}+3 r_{p}^{3 / 2}}\right\} .
$$

This criterion is a little less precise than the criterion obtained above, (13), but allows us to map the upper limit for optimality over a wide range of initial conditions. FIGURE 7 shows contour plots for equality in (14). At each value of $r_{p}$ and $r_{a}$, 


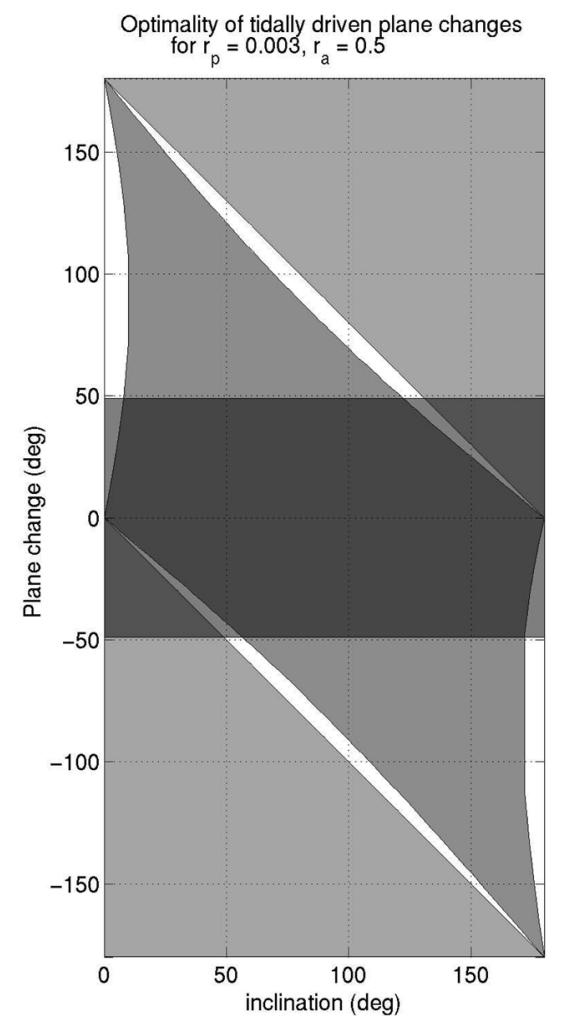

FIGURE 6. A bound on the domain of optimality of one impulse maneuvers.

third-body driven plane changes are optimal, if realizable, for $|\Delta i|$ greater than the value indicated on the graph. Note that this criterion should be applied to the value $r_{a_{\max }}$ chosen, which should lie between 0.4 and 0.7 , depending on the maximum transfer time allowed.

At this point it would seem that a lower bound on conditions for which oneimpulse plane changes are optimal could be obtained by the same method. However, a closing relation, $r_{a}\left(\Delta i_{\max }\right)$, is missing in order to obtain such a bound. We do not know, a priori, what apoapsis radius is needed to perform a given plane change and the Jacobi constant estimates do not allow us to obtain a meaningful bound. The restrictions $V_{l c} \leq v_{2} \leq V_{\text {escape }}$ does not yield any interesting results.

However, one can use the above results to devise an algorithm that answers completely the question of optimality below the theoretical upper limit obtained above. Instead of comparing the $\Delta V$ values at fixed $\Delta i$, one can compare the $\Delta i$ values realized for a fixed, given $\Delta V$. This is possible because of the $1: 1$ relation between $\Delta i$ and $\Delta V_{1-i m p u l s e}$ on one hand, and the same monotonic dependence of $\Delta i$ and $\Delta V$ on $r_{a}$ for third-body driven plane changes, on the other hand. 
Given $\Delta i_{0}$, we have $\Delta V_{1 \text {-impulse }}$ given by Equation (5). Thus, we can solve for $r_{a}$ in $\Delta V^{ \pm}\left(r_{a}\right)=\Delta V_{1 \text {-impulse, yielding two solutions }}$

where

$$
r_{a}^{ \pm}=\frac{\left(\alpha^{ \pm}\right)^{2} r_{p}}{2-\left(\alpha^{ \pm}\right)^{2}}, \quad \alpha^{ \pm}=\frac{A^{2}-B^{ \pm}}{2 V_{l c}\left(A-r_{p} \cos i\right)}
$$

$$
\begin{gathered}
A=\Delta V_{1-\text { impulse }}-r_{p} \cos (i+\Delta i)+2 V_{l c} \\
B^{+}=4 r_{p}^{2} \cos ^{2}(i+\Delta i), \quad B^{-}=r_{p}^{2} \cos ^{2}(i+\Delta i)-r_{p}^{2} \cos ^{2}(i) .
\end{gathered}
$$

Problem (6) can now be solve numerically with $r_{a}^{ \pm}$, resulting in $\Delta i^{ \pm}$to be compared with $\Delta i_{0}$.

Algorithm

Our algorithm is formulated here for $\Delta i_{0} \geq 0$. A similar algorithm can be written for $\Delta i_{0} \leq 0$.

- Given $r_{p}, i$, and $\Delta i_{0}$, solve for $\Delta V_{1 \text {-impulse }}$ using (5).

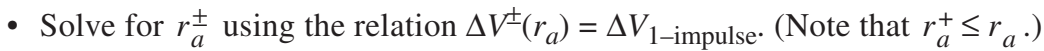

- Solve the optimization problem (6) with $r_{p}, r_{a}^{ \pm}, i$ to obtain $\Delta i^{ \pm}$. (Note that $\Delta i^{+} \leq \Delta i^{-}$.)

- compare:

If $\Delta i_{0} \geq \Delta i^{-}$, then one-impulse transfers are optimal.

If $\Delta i_{0} \leq \Delta i^{-}$, then third-body driven plane changes are optimal.

If $\Delta i^{+}<\Delta i_{0}<\Delta i^{-}$, then use a dichotomy on $r_{a}$ to solve exactly for $\Delta V_{\text {third-body. }}$

Note that when $\Delta i^{+}<\Delta i_{0}<\Delta i^{-}$, one needs to solve exactly for $\Delta V_{\text {third-body }}$ to decide the optimality by comparison with $\Delta V_{1 \text {-impulse }}$ In practice, this case covers only a

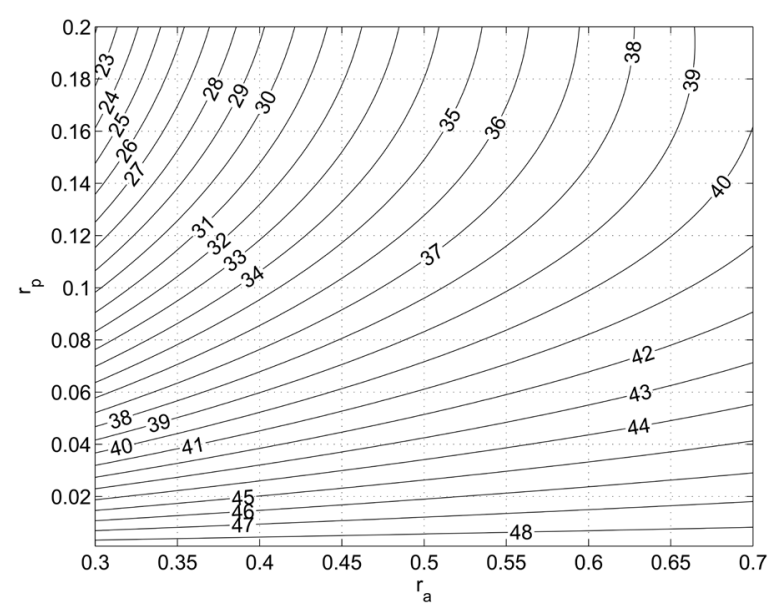

FIGURE 7. Analytic bound for the optimality of third-body driven plane changes. 
very thin strip of initial conditions and generally, the above algorithm requires only solving problem (6) once or twice per set of initial conditions to settle the question.

\section{Optimal $\Delta V$}

In the region of optimality of third-body driven plane changes, the optimal $\Delta V$ needed to perform a given plane change $\Delta i_{0}$ is determined by solving problem (7). Although this problem could, a priori, be solved by any optimizer routine, an easier method consists of reducing the solution of (7) to a sequence of problems (6), for which an algorithm has already been derived. ${ }^{3}$

Indeed, it has been shown numerically ${ }^{3}$ that, for any given initial conditions $r_{p}$ and $i$, the magnitudes of the extrema of third-body driven plane changes increase with $r_{a}$. Therefore, a dichotomy between $r_{p}$ and $r_{a_{\max }}$ can be performed to converge toward the desired plane change value $\Delta i_{0}$. Since we imposed $r_{a_{\max }}<0.7$, the algorithm will converge towards a desired plane change value in less than seven iterations for a error on $r_{a}$ of \pm 0.005 .

Although this algorithm is rather intense computationally, it has the advantage of not requiring any initial guess to converge.

\section{CONCLUSION}

The optimality of third-body driven plane changes has been investigated both analytically and numerically. In particular, an analytic estimate of the limit of optimality has been derived, showing that third-body driven plane changes are, indeed, more optimal than one-impulse maneuvers for a large domain of initial conditions and plane changes larger than about $40^{\circ}$. The computation of the optimal $\Delta V$ needed to perform a given plane change has been reduced to the solution of a simpler optimization problem that does not require any initial condition to converge toward the global extremum. Limitations of third-body driven plane changes have been found because of the restrictions imposed by the natural dynamics. It seems that these limitations could be overcome by using artificial propulsion systems in conjunction with the use of cost free third-body forces, resulting in additional optimality criteria. Future research is needed to answer such possibilities.

\section{REFERENCES}

1. Сновотоv, V.A., Ed. 1996. Orbital Mechanics, 2nd edit. AIAA Education Series.

2. Villac, B.F., D.J. Scheeres, L.A. D’Amario \& M.D. Gumann. 2001. The effect of tidal forces in orbit transfers. Proceedings AAS/AIAA Spaceflight Mechanics Meeting, Santa Barbara, CA.

3. Villac, B.F. \& D.J. ScheERES. 2003. New class of optimal plane change maneuvers. J. Guid. Control Dynam. 26(5): 750-757.

4. Scheeres, D.J., M.D. Guman \& B. VillaC. 2001. Stability analysis of planetary satellite orbiters: application to the Europa Orbiter. J. Guid. Control Dynam. 24(4): 778-787 